\title{
Aspectos metodológicos críticos en las evaluaciones económicas de salud en el contexto cubano
}

\author{
Critical methodological aspects of the health economic \\ assessments in the Cuban setting
}

\author{
Anaí García Fariñas; Ana María Gálvez González ${ }^{\mathrm{II}}$; José Félix García \\ Rodríguez $^{\text {III }}$ \\ ${ }^{\mathrm{I}}$ Investigador Agregado. Asistente. Instituto de Farmacia y Alimentos. Universidad \\ de La Habana. La Habana, Cuba. \\ ${ }^{\mathrm{II}}$ Doctor en Ciencias de la Salud. Investigador y Profesor Titular. Escuela Nacional \\ de Salud Pública. La Habana, Cuba.

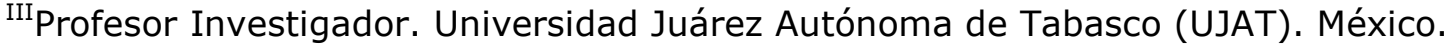

\section{RESUMEN}

Diversos factores participan como determinantes en el aumento sostenido de los costos de atención de la salud, lo que ha obligado a los gobiernos de todos los países del mundo a priorizar la asignación de los recursos disponibles entre diversas opciones de gasto en salud. Ante esta situación es de suma importancia lograr una mayor eficiencia en la aplicación de los recursos existentes. En este artículo se presentan los pasos básicos para la realización de evaluaciones económicas en salud, escasas aún en Cuba, con el objetivo de contribuir a su mejor comprensión. Se profundiza en dos de las acciones específicas de este tipo de estudio: la identificación de opciones de comparación y el análisis de resultados. A pesar del paso de avance dado en el campo metodológico con la Guía de Evaluación Económica en Salud cubana todavía se debe insistir en el rigor metodológico de este tipo de estudios.

Palabras clave: Evaluación económica, tecnologías sanitarias, metodología, guía metodológica. 


\section{ABSTRACT}

A number of factors are involved as determinants in the sustained increase of health care costs, all of which has forced the governments worldwide to prioritize the allocation of the available resources among several health expenditure choices. In the face of this situation, it is extremely important to achieve higher efficiency in the use of existing resources. This paper presented the basic steps to make health economic assessments, still very limited in Cuba, in order to contribute to a better understanding. It delved into two specific actions of this type of study, that is, identification of comparison options and analysis of results. Despite the step forward represented by the Cuban Health Economic Assessment Guideline in the methodological field, it is necessary to insist on the methodological rigor that this type of studies must have.

Key words: Economic assessment, health technologies, methodology, methodological guideline.

\section{INTRODUCCIÓN}

El aumento sostenido en los costos de atención de la salud constituye una preocupación mundial. Se han descrito diversos factores como determinantes de este fenómeno, entre los cuales se citan: a). Cambios demográficos que se traducen en poblaciones de edad más avanzada cuya atención exige servicios más caros y especializados. b). Una transición epidemiológica caracterizada por la prevalencia de padecimientos crónicos o no transmisibles como causas de enfermedad y muerte, y minusvalías propias de la modernidad y los nuevos estilos de vida. Ello asociado a la presencia de enfermedades propias de la pobreza, la reemergencia de enfermedades infecciosas y la emergencia de nuevos padecimientos. c). Adelantos inusitados en la tecnología médica, que conducen a sistemas de diagnóstico más complejos, a su uso intensivo, y a la ampliación del alcance de la atención médica. d). Mayores expectativas de los usuarios y de los profesionales de la salud, lo que incide en un incremento de la demanda sanitaria.

Lo anterior ha obligado a los gobiernos de todos los países del mundo, tanto pobres como ricos, a priorizar la asignación de los recursos disponibles entre diversas opciones de gasto en salud. En términos económicos, esto supone un costo de oportunidad, ya que cualquier toma de decisión implicará un sacrificio económico y social, puesto que una vez destinado un recurso para un fin, no podrá ser utilizado nuevamente. Ante ello, varios economistas han señalado que lograr una mayor eficiencia en la aplicación de los recursos existentes debería ser, entre otros, uno de los criterios básicos de priorización de necesidades en salud. ${ }^{1}$ En este contexto se insertan los estudios de evaluación económica de tecnologías sanitarias. De la misma manera, la literatura sobre evaluación económica en atención a la salud ha aumentado progresivamente, y cada vez son más los estudios de naturaleza multidisciplinaria. ${ }^{2}$ 
En la actualidad todavía son pocos los países que emplean la información obtenida de estos estudios como fundamento para la toma de decisiones. Las brechas entre las investigaciones y la consideración de sus resultados para la toma de decisiones han estado determinadas, entre otros factores, por la falta de consenso en determinados aspectos metodológicos y por la mala calidad de muchos de los estudios realizados. A este respecto los estudiosos de este tema se han pronunciado a nivel mundial, se han aunado los esfuerzos en aras de aportar herramientas que ayuden a salvar este escollo. Entre otras acciones, se han desarrollado guías metodológicas, tanto a nivel de países, ${ }^{3}$ como de organizaciones internacionales. ${ }^{4,5}$ Cuba también forma parte de este esfuerzo. En el año 2004 se publicó la Guía Metodológica para la Evaluación Económica en Salud en Cuba. ${ }^{6}$ No obstante, todavía son escasas las evaluaciones económicas completas que se realizan en el país, al tiempo que subsisten errores de diseño en no pocas de ellas. Entre las acciones que se desarrollan con el fin de subsanar estos errores está el continuo perfeccionamiento de la Guía de Evaluaciones Económicas Cubana. En ese marco se inserta el presente artículo cuyo objetivo es profundizar en dos aspectos de la metodología de evaluación económica en salud que suelen no ser bien tratados, con el fin de contribuir a su mejor entendimiento y con ello, a una mejor práctica investigativa.

\section{Pautas metodológicas básicas}

Los diferentes trabajos metodológicos desarrollados internacionalmente, ${ }^{7-9}$ coinciden en que la ejecución de un estudio del tipo evaluación económica, cualquiera que sea, debe al menos cumplir los siguientes pasos lógicos:

1. Definir el problema a estudiar.

2. Fijar los objetivos que se desean alcanzar.

3. Identificar y describir las opciones.

4. Establecer la perspectiva del análisis.

5. Identificar y medir los recursos necesarios para cada opción.

6. Identificar y medir los resultados de cada opción.

7. Analizar la relación recursos resultados de cada opción.

8. Realizar el ajuste temporal.

9. Realizar el análisis de sensibilidad.

10. Presentar los resultados.

Algunos de estos pasos son comunes a cualquier investigación que siga el método científico como por ejemplo, la definición del problema y la identificación de los objetivos que se persiguen lograr, sin embargo aspectos tales como la identificación y descripción de opciones, el definir o establecer la perspectiva del estudio, el análisis de los resultados y el ajuste temporal, son elementos propios y fundamentales de las evaluaciones económicas. En este artículo se profundizará en dos de los esos aspectos distintivos: la identificación y descripción de las alternativas y el análisis de los resultados. 


\section{De la identificación y descripción de las opciones}

La evaluación económica implica la comparación de opciones, de aquí que una vez esclarecido los objetivos estas deben identificarse y describirse. Este resulta uno de los aspectos más controversiales, pues el número y tipo de opciones que se seleccionen condicionarán los resultados finales del estudio. Se puede entender, entonces, la necesidad el esclarecer qué se considera "opción". Opciones son diferentes tecnologías dirigidas a un mismo objetivo que se diferencian sustancialmente en sus naturalezas organizacionales y tecnológicas o ambas. Las diferentes opciones deben permitir alcanzar un objetivo común de ahí que al realizar una evaluación económica de ellas se asume la posibilidad de sustitución recíproca, de hecho los resultados finales de la evaluación económica contribuyen a soportar la decisión de asignar determinados recursos a una u otra opción.

En la práctica cotidiana las principales deficiencias en este respecto son: no siempre están incluidas todas las opciones relevantes, además los investigadores suelen confundir opciones con diferentes etapas dentro de una misma solución, o considerar como opciones aquellas soluciones dirigidas a grupos de individuos con características diferentes. Por ejemplo, si las condiciones para la valoración de un tratamiento de naturaleza quirúrgica es la resistencia farmacológica, tal resistencia no debe ser considerada opción a la primera. Una evaluación económica que comparara ambas opciones arrojaría resultados no fiables pues falsamente se estaría suponiendo que a todos los individuos se les puede tratar con una u otra opción cuando en realidad no es cierto.

El conjunto de opciones a considerar en una evaluación económica debe ser exhaustivo y las opciones pueden ser mutuamente excluyentes o combinaciones de estas. En general, es necesario ser cuidadoso en la identificación de todas las opciones relevantes de forma tal que se evite el sesgo de selección. Entre las opciones se aconseja considerar desde la conocida opción nula o "no hacer nada" pasando por la opción que se esté desarrollando en la práctica hasta la mejor forma de solucionar el problema objeto de estudio informada en la literatura. En todos los casos, la selección de las opciones deberá fundamentarse sobre la base de su eficacia o efectividad para resolver el problema que dio origen a la evaluación económica. Una opción de eficacia/efectividad dudosa no debe ser considerada en una evaluación económica. De la misma manera, la reciente atención prestada al análisis de costobeneficio, requiere una metodología explicita de valoración de la vida humana, como requisito indispensable para calcular debidamente los beneficios previstos. ${ }^{10}$

\section{Del análisis de los resultados}

Una vez identificados y valorados los resultados en la salud y los recursos, es necesario relacionarlos para comparar la eficiencia de cada opción. Para esto pueden desarrollarse dos tipos de análisis: comparar los valores medios de las razones costos resultados de cada opción en estudio y realizar el análisis incremental donde se comparan simultáneamente los costos y resultados de las opciones. La comparación se realiza entre pares de opciones. La razón costo resultado incremental, se obtiene de dividir el incremento de los costos (costo de la opción A - costo de la opción B) entre el incremento en los resultados (resultados de la opción $A$ - resultados de la opción B).

Una vez obtenido el costo resultado medio (costo efectividad o costo utilidad), el análisis suele dirigirse a favorecer la opción con menor costo resultado como indicativo de su eficiencia. No obstante, cómo saber si el costo por unidad de resultado de la supuesta opción más eficiente es demasiado alto o bajo para 
determinado contexto. Ante esta preocupación algunos investigadores han tomado como referencia el valor del PIB per cápita y han fundamentado el análisis en que cualquier opción que represente un costo por unidad de resultado mayor que la producción media del país no debería considerase como viable. De cualquier manera, debe encontrarse un referente objetivo propio del lugar donde se realiza el estudio para valorar globalmente si las opciones son costo efectivas o no.

Expresiones como que la opción estudiada es costo efectiva ante el argumento de que la salud no tiene precio no deberían ser utilizadas en el contexto de una evaluación económica.

A manera de conclusiones puede afirmarse que en Cuba se dio un paso de avance en lo relativo al campo metodológico con la creación de la Guía para Evaluaciones Económicas en Salud, la cual, durante los últimos cinco años, ha sido ampliamente usada especialmente por los alumnos de la Maestría en Economía de la Salud. No obstante, todavía son escasos estos estudios y más aún el empleo de la información que de ellos se deriva para la toma de decisiones. Para potenciar el uso de las evaluaciones económicas por las autoridades sanitarias cubanas se deberá no sólo incrementar el número de investigaciones sino que se deberá ser celoso en el rigor metodológico de los estudios que se realicen.

\section{REFERENCIAS BIBLIOGRÁFICAS}

1. Madueño-Dávila M, Sanabria-Montañes C. Estudio de oferta de los servicios de salud en el Perú y el análisis de brechas (2003-2020). Bethesda, Maryland, U.S: Agency for Internacional Development (USAID); 2003.

2. Drummond M. Métodos para la evaluación económica. Madrid: Diáz de Santos; 1991.

3. Niessen LW, Grijseels E, Koopmanschap M, Rutten F. Economic analysis for clinical practice the case of 31 national consensus guidelines in the Netherlands. J Evaluation Clinil Prac. 2007;13:68-78.

4. World Health Organization (WHO). Guide to cost-effectiveness analysis. Switzerland: WHO; 2003.

5. Salazar L, Jackson S, Shiell A, Rice M. Guía de Evaluación Económica en Promoción de Salud. Washington, D.C: OPS;2007.

6. Gálvez AM. Guía Metodológica para la Evaluación Económica en Salud en Cuba. Rev Cubana Salud Pública. 2004;30(1):37-54.

7. Badía X, Rovira J. Evaluación Económica de Medicamentos, un instrumento para la toma de decisiones en la práctica clínica y la política sanitaria. Barcelona: Luzán; 1994.

8. Sacristán JA, Ortún V, Rovira J, Prieto L, García-Alonso F. Evaluación económica en medicina. Med Clin (Barc). 2004;122 (10):379-82.

9. Drummond M, O'Brien BJ, Stoddart GL, Torrance GW. Métodos para la evaluación económica de los programas de asistencia sanitaria. 3ra ed. Madrid: Díaz de Santos; 2006. 
10. Organización Panamericana de la Salud. Investigaciones sobre servicios de salud. Una antología. OMS-OPS. Publicación Científica No. 534. Washington, D.C.: OPS; 1992.

Recibido: 8 de octubre de 2009.

Aprobado: 26 de febrero de 2010.

Anai García Fariñas. Instituto de Farmacia y Alimentos (IFAL). Calle 222 y 27. La Lisa. La Habana, Cuba.

E-mail:alastor@infomed.sld.cu 